\title{
Intenção de abandono profissional entre professores: o papel dos estressores ocupacionais
}

\author{
Mary Sandra Carlotto' \\ Sheila Gonçalves Câmaral" \\ Michelle Engers Taube de Oliveira"l

RESUMO

A intenção de abandonar a profissão é um importante preditor do abandono profissional, com repercussões negativas para a qualidade do ensino, desempenho escolar e comunidade em geral. O objetivo do estudo foi identificar o poder preditivo dos estressores ocupacionais para a tendência ao abandono profissional em professores. Participaram do estudo 376 professores de escolas municipais de uma cidade da região metropolitana de Porto Alegre. Como instrumentos de avaliação foram utilizados um questionário de dados sociodemográficos e laborais, uma escala de avaliação de tendência ao abandono e um questionário de estressores ocupacionais docentes. Os resultados, obtidos por meio da análise de regressão linear, apontaram como preditores do abandono profissional os estressores multiplicidade de papéis a desempenhar, conciliar trabalho e lazer e a relação com alunos. $O$ estudo sugere possíveis intervenções como forma preventiva, assim como indica possibilidades de novos estudos.

\section{PALAVRAS-CHAVE}

intenção de abandono profissional; estresse ocupacional; professores.

'Universidade do Vale do Rio dos Sinos, São Leopoldo, RS, Brasil.

"Universidade Federal de Ciências da Saúde de Porto Alegre, Porto Alegre, RS, Brasil.

"'Universidade do Vale do Taquari, São Leopoldo, RS, Brasil. 


\title{
THE INTENTION TO LEAVE THE PROFESSION AMONG TEACHERS: THE ROLE OF OCCUPATIONAL STRESSORS
}

\begin{abstract}
The intention to leave the profession is an important predictor of professional abandonment, with negative repercussions on the quality of teaching, school performance and community in general. The objective of the study was to identify the predictive power of occupational stressors for the tendency to drop out in teachers. A total of 376 municipal school teachers from a city in the metropolitan region of Porto Alegre participated in the study. As instruments of evaluation, a questionnaire of sociodemographic and labor data, a scale of evaluation of abandonment tendency and a questionnaire of occupational stressors were used. The results, obtained through the linear regression analysis, pointed out as predictors of professional abandonment the stressors multiplicity of roles to play, to reconcile work and leisure and the relationship with students. The study suggests possible interventions as preventive as well as indicates possibilities of new studies.
\end{abstract}

KEYWORDS

intention to leave the profession; occupational stress; teachers.

\section{INTENCIÓN DE ABANDONO PROFESIONAL ENTRE PROFESORES: EL PAPEL DE LOS ESTRESORES OCUPACIONALES}

\section{RESUMEN}

La intención de abandonar la profesión es un importante predictor del abandono profesional, con repercusiones negativas para la calidad de la enseñanza, desempeño escolar y comunidad en general. El objetivo del estudio fue identificar el poder predictivo de los estresores ocupacionales para la tendencia al abandono en profesores. Participaron del estudio 376 profesores de escuelas municipales de una ciudad de la región metropolitana de Porto Alegre. Como instrumentos de evaluación se utilizó un cuestionario de datos sociodemográficos y laborales, una escala de evaluación de tendencia al abandono y un cuestionario de estresores ocupacionales docentes. Los resultados, obtenidos por medio del análisis de regresión lineal, apunta como predictores del abandono profesional los estresores multiplicidad de papeles a desempeñar, conciliar trabajo y ocio y la relación con alumnos. El estudio sugiere posibles intervenciones como forma preventiva, así como indica posibilidades de nuevos estudios.

intención de abandono profesional; estrés ocupacional; profesores. 


\section{INTRODUÇÃO}

A rotatividade de professores gerada pelo abandono da profissão é um importante fenômeno global que tem adquirido crescente visibilidade em virtude de suas implicações negativas para a qualidade do ensino, do desempenho escolar e para a comunidade em geral, assim como por seu impacto social e econômico (Lee et al., 2015; Saltini, Vidal e Oliveira-Sobrinho, 2014). Esse fenômeno tem sido substancialmente investigado no que diz respeito à sua prevalência, no entanto os fatores que contribuem para sua ocorrência ainda precisam de maior investigação e debate (Fitchett et al., 2016). A rotatividade dos professores pode ocorrer por mobilidade interna no próprio sistema de ensino ou por abandono definitivo da profissão. No primeiro caso, os professores optam por mudar de escola por motivos pessoais ou profissionais, visando buscar melhores condições de trabalho ou maior comodidade em localização geográfica (Pereira Junior e Oliveira, 2016).

Várias são as causas destacadas pela literatura atribuídas ao abandono profissional de professores. Entre elas podem-se citar a insatisfação no trabalho, o baixo salário, as dificuldades na gestão de relações sociais e de adaptação às inúmeras demandas escolares, a falta de recursos materiais, autonomia e participação na tomada de decisões institucionais (Buchanan et al.,2013), além de dificuldades no controle de sala de aula, as relações conflitantes com pais de alunos, colegas e gestores, a falta de eficácia e a percepção das responsabilidades de ensino como muito intensas (Hong, 2010). Ressaltam-se, ainda, a desvalorização profissional, a indisciplina e a violência dos alunos na escola, o ambiente social negativo de trabalho, a falta de progressão continuada, a pouca participação familiar no trabalho educativo e as más condições de trabalho, entre outras (Lemos, 2009).

O desligamento definitivo da profissão é a etapa final de um processo que inicia com a intenção de abandonar a profissão (Cohen, Blake e Goodman, 2016; Mobley, 1977; Mobley, Horner e Hollingsworth, 1978; Tekleab, Takeuchi e Taylor, 2005). O conceito de intenção de abandono é caracterizado como um processo psicológico, de natureza cognitiva e afetiva, que começa com a avaliação/satisfação do trabalho, alternativas de mercado e custos/benefícios da decisão de saída da profissão ou do local de trabalho (Mobley, 1977). A saída da instituição é a ação final de uma série de mecanismos cognitivos, afetivos e comportamentais, utilizados ao longo de um tempo, que conduzem ao rompimento do vínculo (Dickter, Roznowski e Harrison, 1996). O rompimento total do vínculo é uma decisão difícil, que somente é tomada quando se esgotaram todas as possibilidades possíveis de continuar exercendo a profissão docente de acordo com os princípios e valores do professor (Saltini, Vidal e Oliveira-Sobrinho, 2014).

A intenção de abandono é considerada um importante problema internacional (Vekeman et al., 2016). A preocupação sobre esse fenômeno tem aumentado nos últimos anos tanto por parte de pesquisadores como de organismos internacionais, gestores educacionais e pela própria categoria docente (ILO, 2005; Ingersoll, 2001; Lee et al., 2015; Skaalvik e Skaalvik, 2015; OECD, 2005, 2014; UNESCO, 2016). Tal preocupação decorre do fato de a rotatividade de professores gerar prejuízos no processo de aprendizagem, na formação de equipes coesas e na manutenção das relações com a comunidade escolar, impactando negativamente na qualidade de ensino ofertado à população (Cassettari, Scaldelai e Frutuoso, 2014). 
A perda de professores pela exoneração voluntária traz custos significativos para o sistema educacional em virtude da necessidade de substituição, que gera a replicação dos custos de contratação e formação (Cassettari, Scaldelai e Frutuoso, 2014). A retenção de professores tornou-se um importante problema a ser enfrentado pelos gestores políticos e educacionais, tendo em vista o desequilibrio entre a crescente demanda proveniente do aumento da população em idade escolar, a saída de professores por aposentadoria e a diminuição do interesse em ingressar na carreira docente (Pucella, 2011), principalmente por parte de jovens (OECD, 2005; Tartuce, Nunes e De Almeida,2010) e estudantes em formação (Clement, 2017). Abandonar a profissão implica custos emocionais, financeiros, diminuição do reconhecimento social e da identidade profissional, assim como a perda do tempo, treinamento e investimento financeiro (Carson, Carson e Bedeian, 1995; Eugênio, Souzas e Di Lauro, 2017). Identificar as causas do abandono docente pode subsidiar políticas para a resolução desse problema (Lee et al., 2015; Lee et al., 2017).

O pensamento ou a intenção de abandonar a profissão pode ser considerado um abandono especial, sendo uma das suas formas mais problemáticas, pois representa a desistência do exercício do magistério sem efetivamente concretizar sua saída. Nesse tipo de abandono, o professor comparece à escola, ministra aulas, cumpre as obrigações burocráticas, mas executa essas atividades dentro de um limite que representa o mínimo necessário para manter-se no emprego (Clement, 2017; Lapo e Bueno, 2002). Esse também tem sido denominado de abandono psicológico, o qual funciona como uma estratégia de distanciamento para lidar com as demandas e com a insatisfação no trabalho. As pessoas continuam trabalhando, mas muito abaixo de seu potencial laboral e apresentando problemas de desempenho e qualidade de trabalho (Maslach e Goldberg, 1998).

A intenção do abandono docente - fenômeno de caráter mundial — possui variações importantes, de acordo com o contexto social e organizacional no qual o exercício docente é realizado. Países como Austrália e Estados Unidos pesquisaram a tendência ao abandono da profissão e identificaram que $45 \%$ dos professores australianos consideram deixar a profissão em 10 anos, e cerca de $35 \%$ dos professores estadunidenses pretendem abandonar a profissão em 3 anos, 50\% em 10 anos e 10\% efetivamente a abandonam anualmente (AEU, 2008; Boyd et al., 2008). No Chile, nos anos 2013 e 2014, 7\% a 8\% solicitam saída antecipada do sistema de ensino, ou seja, antes da idade para a aposentadoria. Entre os ingressantes no sistema, $40 \%$ dos professores abandonam a profissão até o quinto ano do exercício profissional (Centro de Estudios Mineduc, 2015). No Brasil, levantamento realizado por Fleuri (2015), com base no Teaching and Learning International Survey (TALIS), realizado em 2013 com a participação do Brasil (OECD, 2014), identificou que $10 \%$ dos professores brasileiros consideravam abandonar a profissão.

Altos níveis de estresse têm sido relacionados à decisão de abandonar a profissão (Clement, 2017). A maioria dos professores que pensa ou abandona a profissão antes da aposentadoria o faz por desgaste físico e psicológico (Ingersoll, 2001), e isso em virtude da exposição frequente a estressores ocupacionais (Ingersoll, 2012; Wang et al., 2017). O estresse em professores também está relacionado com maiores níveis de insatisfação, absenteísmo e a probabilidade de abandono da profissão (Jeyaraj, 2013; Koros, Momanyi e Chakua, 2018).

Nesse sentido, pesquisar e ampliar o conhecimento sobre os fatores de desgaste é importante para subsidiar gestores na implantação de políticas de gestão 
que sejam capazes de identificar os riscos de abandono e mudar as condições que fundamentam a decisão de permanecer ou abandonar a profissão (Cassettari, Scaldelai e Frutuoso, 2014; Horvath, Goodell e Kosteas, 2018; Lindqyist, Nordänger e Carlsson, 2014). Assim, pelo exposto, o estudo teve como objetivo identificar o poder preditivo dos estressores ocupacionais para a tendência ao abandono em professores.

\section{MÉTODO}

\section{PARTICIPANTES}

Este estudo de corte observacional, analítico e transversal (Grimes e Shulz, 2002) teve como amostra não probabilística 376 professores da educação básica (ensino infantil e fundamental) de escolas públicas municipais de uma cidade da região metropolitana de Porto Alegre, Rio Grande do Sul, Brasil. Foram incluídos no estudo os professores que trabalhavam exclusivamente em uma escola e não possuíam outra atividade profissional.

A maioria dos participantes pertencia ao sexo feminino (95,7\%), referiu ter uma união estável $(76,3 \%)$, possuía filhos $(76,7 \%)$ e formação em nível de pós-graduação (69,7\%). A média de idade foi de 44 anos ( $\mathrm{DP}=8,3$; amplitu$\mathrm{de}=18$ a 66) e a de tempo de experiência docente foi de 18,3 anos (DP $=10$ amplitude $=18$ a 44). A carga horária de trabalho semanal variou de 12 a 60 horas, sendo a média de 33 horas $(\mathrm{DP}=10)$.

\section{INSTRUMENTOS} cáveis:

Os dados foram coletados por meio dos seguintes instrumentos autoapli-

- Questionário de dados sociodemográficos (sexo, idade, situação conjugal, filhos, formação) e laborais (carga horária, tempo de experiência docente);

- Tendência ao abandono: subescala da bateria de avaliação de riscos psicossociais (Unidad de Investigación Psicosocial de la Conducta Organizacional - UNIPSICO) de Gil-Monte (2005), traduzida e adaptada para o presente estudo. A subescala é constituída por quatro itens (alfa $=$ 0,83 ; exemplo: eu penso em abandonar minha profissão) que são avaliados com uma escala de frequência de cinco pontos (0 "nunca"; 1 "poucas vezes"; 2 "algumas vezes"; 3 "frequentemente"; 4 "todos os dias");

- Questionário de estresse ocupacional docente baseado na literatura sobre estressores ocupacionais em professores (Carlotto e Pallazzo, 2006) e relatados em intervenção realizada também com professores (Carlotto, 2014).

O instrumento avalia a percepção de 21 fatores de estresse, a saber:

1. carga horária mensal;

2. quantidade de alunos por classe;

3. carga de trabalho diária;

4. diversidade de atividades diárias; 
5. multiplicidade de papéis a desempenhar;

6. realização de atividades burocráticas;

7. ambiente/clima social de trabalho;

8. relação com colegas;

9. relação com direção;

10. relação com alunos;

11. relação com pais/responsáveis;

12. atender às expectativas de pais/responsáveis e sociedade;

13. relação com a gestão municipal;

14. pouca participação nas decisões institucionais;

15. condições físicas de trabalho;

16. falta de recursos materiais para o trabalho;

17. organização/forma com que seu trabalho é realizado;

18. contínua necessidade de atualização;

19. dificuldade em conciliar trabalho e família;

20. dificuldade em conciliar trabalho e lazer;

21. deslocamento ida/volta do trabalho.

Os itens são avaliados com uma escala de cinco pontos (0 "nada estressante"; 1 "pouco estressante"; 2 "relativamente estressante"; 3 "bastante estressante"; 4 "altamente estressante"). A escala apresentou um valor de alfa $=0,94$.

\section{PROCEDIMENTOS}

Após explanação dos objetivos e autorização da Secretaria Municipal de Educação, o objetivo do estudo foi apresentado para os diretores das escolas para obtenção do apoio e definição da logística de coleta de dados. Os instrumentos foram aplicados em reunião de professores, sendo recolhidos ao término do preenchimento. Foi esclarecido aos professores tratar-se de uma pesquisa sem quaisquer efeitos avaliativos individuais e/ou institucional, com respostas anônimas e confidenciais. Foram realizados os procedimentos éticos conforme resolução 466 do Conselho Nacional de Saúde do Brasil (CNS). A pesquisa possui aprovação do Comitê de Ética da Pontifícia Universidade Católica do Rio Grande do Sul (PUC-RS).

O banco de dados foi digitado e posteriormente analisado em pacote estatístico PASW, versão 17 (SPSS/PASW Inc., Chicago, IL). Para identificar os preditores, foi empreendida uma análise de regressão linear múltipla, método Stepwise, após a realização de seus pressupostos, de acordo com Field (2009). Foi verificada a ausência de multicolinearidade, pois todos os valores das correlações ficaram abaixo de 0,763, os valores de Variance Inflation Factor (VIF) situaram-se abaixo de $4(1,457)$, e os valores de tolerância foram inferiores a $1(0,965)$. A análise do coeficiente de Durbin-Watson identificou valor próximo a $2(1,974)$, indicando a independência da distribuição e a não correlação dos resíduos. A distância de Cook apresentou valor inferior a $1(0,003)$, indicando não existir preditores atípicos e um adequado ajuste dos modelos. A análise assumiu como variável dependente a tendência ao abandono e como variáveis independentes os estressores ocupacionais. As variáveis idade, nível 
de formação e tempo de experiência foram controladas. Para a inclusão das variáveis dependentes no modelo de regressão linear múltipla, foram selecionadas aquelas que obtiveram valor $\mathrm{p} \leq 0,20$ na análise de correlação de Pearson (todos os 21 estressores). Os resultados foram definidos como estatisticamente significativos a um $\mathrm{p} \leq 0,05 . \mathrm{Na}$ análise de regressão, a magnitude do efeito foi obtida pelos coeficientes de regressão padronizados calculados para o modelo final (Field, 2009).

\section{RESULTADOS}

Os resultados obtidos indicam que o maior índice médio foi encontrado no estressor quantidade de alunos por classe e o menor no estressor relação com colegas. Os valores de assimetria e curtose obtidos, tanto na variável dependente quanto nas independentes, permitem afirmar que, em geral, estas apresentam uma distribuição normal. Os valores mais elevados de assimetria e de curtose foram identificados nas variáveis relação com a direção $(A s=1,60 ; \mathrm{Cu}=2,56)$ e relação com colegas $(\mathrm{As}=1,29 ; \mathrm{Cu}=2,10)$ (Tabela 1$)$.

Tabela 1 - Estatísticas descritivas das variáveis em estudo.

\begin{tabular}{l|c|c|c|c}
\hline Variáveis & Variação & M(DP) & As & Cu \\
\hline Variável dependente & & & & \\
\hline Tendência ao abandono & $(0-4)$ & $1,69(0,83)$ & 0,42 & $-0,53$ \\
\hline Variáveis independentes & & & & \\
\hline Carga horária mensal & $(0-4)$ & $1,58(1,15)$ & 0,26 & $-0,74$ \\
\hline Quantidade de alunos por classe & $(0-4)$ & $2,28(1,28)$ & $-0,17$ & $-1,09$ \\
\hline Carga de trabalho diária & $(0-4)$ & $1,63(1,11)$ & 0,27 & $-0,75$ \\
\hline Diversidade de atividades diárias & $(0-4)$ & $1,67(1,10)$ & 0,35 & $-0,67$ \\
\hline Multiplicidade de papéis a desempenhar & $(0-4)$ & $2,14(1,27)$ & $-0,16$ & $-1,07$ \\
\hline Realização de atividades burocráticas & $(0-4)$ & $2,02(1,27)$ & $-0,18$ & $-0,91$ \\
\hline Ambiente/clima social de trabalho & $(0-4)$ & $1,38(1,11)$ & 0,79 & 0,19 \\
\hline Relação com colegas & $(0-4)$ & $0,89(0,90)$ & 1,29 & 2,10 \\
\hline Relação com direção & $(0-4)$ & $1,75(0,95)$ & 1,60 & 2,56 \\
\hline Relação com alunos & $(0-4)$ & $1,56(1,11)$ & 0,51 & $-0,30$ \\
\hline Relação com pais/responsáveis & $(0-4)$ & $1,29(1,00)$ & 0,61 & 0,23 \\
\hline Atender às expectativas de pais/responsáveis e sociedade & $(0-4)$ & $1,68(1,08)$ & 0,38 & $-0,43$ \\
\hline Relação com a gestão municipal & $(0-4)$ & $2,01(1,31)$ & 0,06 & $-1,10$ \\
\hline Pouca participação nas decisões institucionais & $(0-4)$ & $1,77(1,39)$ & 0,42 & $-0,77$ \\
\hline Condições físicas de trabalho & $(0-4)$ & $1,61(1,13)$ & 0,43 & $-0,51$ \\
\hline Falta de recursos materiais para o trabalho & $(0-4)$ & $1,87(1,24)$ & 0,15 & $-1,07$ \\
\hline Organização/forma com que seu trabalho é realizado & $(0-4)$ & $1,26(0,98)$ & 0,56 & $-0,57$ \\
\hline Contínua necessidade de atualização & $(0-4)$ & $1,35(1,01)$ & 0,61 & 0,06 \\
\hline Conciliar trabalho e família & $(0-4)$ & $1,62(1,19)$ & 0,27 & $-0,86$ \\
\hline Conciliar trabalho e lazer & $(0-4)$ & $1,64(1,20)$ & 0,20 & $-0,89$ \\
\hline Deslocamento ida/volta do trabalho & $(0-4)$ & $1,42(1,22)$ & 0,33 & $-1,00$ \\
\hline & & & &
\end{tabular}

M: média; DP: desvio padrão; As: assimetria; Cu: curtose.

Fonte: dados coletados na pesquisa.

Elaboração das autoras 
A Tabela 2 apresenta os resultados da análise de regressão linear que considerou a tendência ao abandono como variável dependente e os estressores ocupacionais como variáveis independentes. A análise dos preditores evidenciou um modelo explicativo constituído por três estressores ocupacionais que, conjuntamente, explicaram $38,4 \%$ da variância da tendência ao abandono. As variáveis idade, nível de formação e tempo de experiência foram utilizadas para controlar a inserção das demais, no entanto nenhuma apresentou associação significativa. Quanto à importância relativa estandartizada das variáveis no modelo, conciliar trabalho e lazer apresentou o maior peso $(\beta=0,350)$, seguida de multiplicidade de papéis a desempenhar $(\beta=0,253)$ e relação com alunos $(\beta=0,161)$ (Pardo Merino e Ruiz Díaz, 2002). O conjunto de variáveis revela que quanto maior a percepção de estresse de ter que conciliar o trabalho com o lazer, ter que desempenhar múltiplos papéis e a relação com alunos, maior é a tendência ao abandono.

Os resultados revelam uma elevada magnitude de efeito considerando que o valor de $\mathrm{R}^{2}$ se situou entre 0,25 e 0,50 , de acordo com os parâmetros utilizados por Field (2009). Assim, os valores obtidos indicam que as relações encontradas possivelmente também estarão presentes na população-alvo de professores.

Tabela 2 - Modelo da análise de regressão linear múltipla da tendência ao abandono.

\begin{tabular}{c|c|c|c|c|c|c|c|c|c}
\hline & & $\mathrm{R}$ & $\mathrm{R}^{2}$ & $\begin{array}{c}\mathrm{R}^{2} \\
\text { Ajustado }\end{array}$ & $\mathrm{B}$ & $\mathrm{SE}$ & $\beta$ & $\mathrm{t}$ & $\mathrm{p}$ \\
\hline \multirow{4}{*}{ Controles } & Idade & 0,014 & 0,000 & $-0,003$ & 0,011 & 0,005 & 0,095 & $-0,922$ & 0,056 \\
\cline { 2 - 10 } & Niv. Form. & 0,114 & 0,013 & 0,007 & 0,071 & 0,076 & 0,043 & 0,936 & 0,350 \\
\cline { 2 - 10 } & Tempo exp. & 0,122 & 0,015 & 0,005 & $-0,001$ & 0,004 & $-0,018$ & $-0,373$ & 0,709 \\
\hline \multirow{3}{*}{ Preditores } & $\mathrm{CTL}$ & 0,545 & 0,297 & 0,288 & 0,263 & 0,040 & 0,350 & 6,537 & $0,001^{*}$ \\
\cline { 2 - 10 } & $\mathrm{MP}$ & 0,603 & 0,364 & 0,353 & 0,191 & 0,041 & 0,253 & 4,664 & $0,001^{*}$ \\
\hline \multirow{2}{*}{ Modelo F } & $\mathrm{RA}$ & 0,619 & 0,384 & 0,371 & 0,142 & 0,045 & 0,161 & 3,140 & $0,002^{*}$ \\
\hline
\end{tabular}

Nota: ${ }^{\text {p}}<0,01$; R: coeficiente de correlação múltipla; R2: coeficiente de determinação; R2 Adjusted: coeficiente de determinação ajustado; B: coeficiente de regressão não estandartizado; SE: desvio-padrão; $\beta$ : Coeficiente de regressão parcial estandartizado; t: teste t de Sudent; F: teste de igualdade de variâncias; Niv. Form.: nível de formação; Tempo exp.: tempo de experiência; CTL: conciliar trabalho e lazer; MP: multiplicidade de papéis a desempenhar; RA: relação com alunos. Fonte: dados coletados na pesquisa.

Elaboração das autoras.

\section{DISCUSSÃO}

Este estudo buscou identificar o poder preditivo dos estressores ocupacionais para a tendência ao abandono em uma amostra de professores. A análise dos preditores revelou que quanto maior a percepção de que são estressores ocupacionais ter que conciliar trabalho e lazer, a multiplicidade de papéis a desempenhar e a relação com alunos, maior é a tendência ao abandono do trabalho.

Ressalta-se que 95,7\% dos participantes eram mulheres. Essa configuração é coerente com os dados apresentados por Carvalho (2018) na comparação da distribuição 
de professores por sexo nos anos de 2009,2013 e 2017. A autora aponta a predominância das mulheres em todas as etapas da educação básica, sendo que na educação infantil e nos anos iniciais do ensino fundamental a participação das mulheres está, em média, em torno de 90\%, e nos anos finais do ensino fundamental, em torno de 69\%. Quanto à Região Sul do Brasil, os dados do Instituto Brasileiro de Geografia e Estatística, de 2015, revelam uma participação das mulheres em torno de 84,5\% (Matijascic, 2017). Nesse sentido, o presente estudo, em consonância com a realidade brasileira e da Região Sul do país, assume que os resultados estão atrelados ao sexo feminino, o qual representa a maioria da população de professores de educação básica.

Quanto ao estressor conciliar trabalho e lazer,o preditor com importância relativa estandartizada, pode-se pensar na atual configuração do trabalho docente caracterizado pela sobrecarga e necessidade de concluir atividades fora do horário de expediente, reduzindo o tempo para atividades não relacionadas ao trabalho. A dificuldade em conciliar atividades de trabalho com tempo para lazer tem sido apontada frequentemente em estudos com professores (Faria e Rachid, 2015; Meira et al.,2014; Silva e Cunha, 2017). A intensificação do trabalho docente, de acordo com Woods (1999), produz uma redução da amplitude de atuação do trabalho, pois as tarefas de alto nível são transformadas em rotinas com maior subserviência a um conjunto de burocracia. Nesse contexto, segundo o autor, há menos tempo para executar o trabalho, para atualização profissional, para o lazer e convívio social, assim como poucas oportunidades de trabalho criativo.

No que diz respeito ao estressor multiplicidade de papéis, os resultados confirmam a literatura, que indica ser esse um dos principais fatores de estresse de professores (Burke, Greenglass e Schwarzer, 1996; Diehl e Carlotto, 2014; Oliveira, 2004), pois a sobrecarga de funções e papéis são percebidas como responsabilidades que não competem ao professor (Shernoff et al.,2011). Nesse alargamento das tarefas e atribuições docentes, além de ensinar, promover conhecimento, incentivar o raciocínio e motivar os alunos para a aprendizagem, os professores devem cuidar da higiene, da nutrição, da saúde e de outras necessidades dos alunos, as quais correspondem a responsabilidades familiares que têm sido transferidas para a escola (Noronha, Assunção e Oliveira, 2008). Os professores são socialmente responsabilizados e sentem-se responsáveis pela formação moral e demandas afetivas, tendo que dividir com a família e com outras agências socializadoras muito mais do que pressupõem as tarefas inerentes à educação formal (Lemos e Novaes, 2015), sem que se sintam preparados para as diversas e complexas situações apresentadas (Diehl e Carlotto, 2014). Na tentativa de atender a essas demandas, os professores assumem as prioridades assistenciais, relegando a aprendizagem e o ensino dos conteúdos a plano secundário (Silva e Abud, 2016). Pode-se pensar que esse distanciamento das atividades essenciais e identitárias da profissão gere sentimentos de frustração e impotência, fazendo com que uma das estratégias para lidar com tais sentimentos seja o pensamento de abandonar a profissão. Uma das principais fontes de angústia entre professores é o sentimento de fracasso em ter que atender às necessidades sociais concomitantemente às demandas prescritas do trabalho docente (Sing e Katoch, 2017).

Quanto ao estressor relação professor-alunos, este tem sido apontado pela literatura como uma das principais fontes de tensão e estresse em professores (Hong, 2010; Leite e Löhr, 2012; Lemos, 2009). Geralmente caracterizado por problemas 
de disciplina, a percepção desse estressor constitui-se em uma constante ameaça para a obtenção dos objetivos pedagógicos (Parsonson, 2012).

Considerando que os professores passam a maior parte do tempo de trabalho na sala de aula, sua relação com o aluno é a fonte mais provável de motivação, assim sua expectativa é de desenvolver relações harmoniosas que lhe proporcionem bem-estar (Spilt, Koomen e Thijs, 2011). A relação professor-aluno é o centro do processo educativo e elemento essencial da função docente, e é nessa relação que emerge a essência da função (Silva e Navarro, 2012). O comportamento inadequado dos alunos, geralmente caracterizado pela indisciplina, propicia nos docentes sentimentos de medo, angústia e tensão emocional (Meira et al., 2014). A tensão das relações gera desgaste psicológico que pode conduzir ao pensamento de abandonar a profissão.

O trabalho docente, embora sua contratação seja de realização em espaço público, na escola, não conta com uma barreira que impeça sua realização no espaço privado, em casa, reduzindo o tempo para atividades pessoais (Faria e Rachid,2015). A organização do trabalho e as condições impostas ao professor demandam considerável parte do seu tempo às atribuições escolares e a sua atualização profissional, limitando seu tempo para atividades de lazer (Meira et al., 2014).

É importante destacar que os estressores que explicam a tendência ao abandono profissional são todos de natureza psicossocial, definidos por Cooper, Dewe e O'Driscoll (2001) como aqueles relacionados à natureza do trabalho e às relações interpessoais. Pode-se pensar que estes expressam um novo cenário do trabalho docente em detrimento de estressores tradicionais, como a falta de recursos materiais e precariedade das condições físicas de trabalho.

Uma das forças do presente estudo é que os dados foram coletados entre professores de escolas públicas, que possuem vínculo único e não dispõem de outra atividade profissional, o que reduz o viés associado a professores que teriam maior possibilidade de realizar um pedido de exoneração sem comprometer sua sobrevivência financeira. Outras dizem respeito à utilização de um consistente modelo teórico dos construtos avaliados, utilização de instrumentos com adequados índices de confiabilidade e a magnitude do efeito das variáveis preditoras do modelo identificado.

O estudo, todavia, apresenta algumas limitações que devem ser consideradas na leitura de seus resultados. Uma delas é seu delineamento transversal, que impede a análise de relações causais. Outra seria a regionalidade da amostra investigada, a qual possui características distintas de outras regiões do país, uma vez que há importantes variações dependendo da região, localização da escola (urbana ou rural) e características socioeconômicas da população escolar (Ingersoll, 2011).

A fim de consolidar e ampliar os resultados obtidos, sugerem-se estudos de delineamento longitudinal misto para avaliar o comportamento e estabilidade do modelo preditivo (Abbad e Carlotto, 2016) e conhecer como se desenvolve o processo de intenção de abandono docente e o impacto dos estressores ocupacionais. Estudos com amostras de diferentes estados e regiões do Brasil seriam importantes para verificar a influência cultural e socioeconômica sobre o abandono docente e os fatores geradores de estresse.

Em relação às implicações para a prática, em termos de prevenção, é importante monitorar a intenção de abandono profissional desde o início da carreira 
docente, desenvolvendo ações com foco no adequado manejo dos estressores ocupacionais. Ações envolvendo toda a comunidade escolar, em seus múltiplos níveis, são fundamentais para diminuir o estresse dos professores. A redução do estresse dos professores ocorre somente quando todos os envolvidos no processo de educação reconhecem as dificuldades e trabalham para aliviar as pressões colocadas aos professores (Clement, 2017).

Aos gestores educacionais sugerem-se ações voltadas para a reconfiguração do conteúdo do cargo, no intuito de proporcionar condições para que o professor desempenhe suas funções atendendo a seus objetivos pedagógicos. Equipes e profissionais especializados de suporte podem contribuir para reduzir a necessidade de o professor desviar-se de seu papel e atribuições docentes. Para o estressor relação professor-aluno e conciliação trabalho-lazer, sugerem-se a realização de grupos de reflexão e troca de vivências para maior instrumentalização sobre o manejo produtivo de conflitos (Leite e Löhr, 2012), manejo de emoções no trabalho e gestão do tempo buscando o equilíbrio entre vida pessoal e trabalho (Carlotto, 2014). Com a falta de professores no horizonte, gestores escolares precisam colocar a estabilidade e a atração de novos docentes como prioridade, sendo as estratégias para reduzir o estresse e o fornecimento de suporte para os professores importantes esforços nessa direção (Clement, 2017).

\section{REFERÊNCIAS}

ABBAD, G. S.; CARLOTTO, M. S. Analyzing challenges associated with the adoption of longitudinal studies in Work and Organizational Psychology. Revista Psicologia: Organizações e Trabalho, Brasília, v. 16, n. 4, p. 340-348, 2016. Disponível em: http://pepsic.bvsalud.org/pdf/rpot/v16n4/v16n4a07.pdf. Acesso em: 12 jan. 2018. http://dx.doi.org/10.17652/rpot/2016.4.12585

AEU - AUSTRALIAN EDUCATION UNION. New Educators Survey 2008 Results and Report. Melbourne, Austrália: AEU, 2008. Disponível em: http://www. setearc.com.au/wp-content/uploads/2013/10/Nesurvey08res.pdf. Acesso em: 12 set. 2017.

BOYD, D.; GROSSMAN, P.; LANKFORD, H.; LOEB, S.; WYCKOFF, J. Who leaves? Teacher attrition and student achievement. National Bureau of Economic Research, Cambrigde, v. 14022, p. I21-J24, maio 2008. Disponível em: http://www. nber.org/papers/w14022.pdf. Acesso em: 5 jan. 2018. http://dx.doi.org/10.3386/w14022 BUCHANAN, J.; PRESCOTT, A.; SCHUCK, S.; AUBUSSON, P.; BURKE, P.; LOUVIERE, J. Teacher retention and attrition: views of early career teachers. Australian Journal of Teacher Education, Sydney: University of Technology, v. 38, n. 3, p. 112-129, 2013. Disponível em: http://ro.ecu.edu.au/cgi/viewcontent.cgi?article=2030\&context=ajte. Acesso em: 12 set. 2017. http://dx.doi.org/10.14221/ajte.2013v38n3.9

BURKE, R.J.; GREENGLASS, E. R.; SCHWARZER, R. Predicting teacher Burnout over time: effects of work stress, social support, and self-doubts and its consequences. Anxiety, Stress and Coping, United Kingdom, v. 9, n. 3, p. 261-275, 1996. Disponível em: http://www.tandfonline.com/doi/abs/10.1080/10615809608249406. Acesso em: 4 mar. 2015. https://doi.org/10.1080/10615809608249406 
CARLOTTO, M. S. Prevenção da síndrome de Burnout em professores: um relato de experiência. Mudanças - Psicologia da Saúde, São Paulo: Universidade Metodista, v. 22, n. 1, p. 31-39, 2014. Disponível em: http://www.bibliotekevirtual. org/index.php/2013-02-07-03-02-35/2013-02-07-03-03-11/934-mud/ v22n01/8672-prevencao-da-sindrome-de-burnout-em-professores-um-relato-deexperiencia.html. Acesso em: 7 abr. 2017. http://dx.doi.org/10.15603/2176-1019/ mud.v22n1p31-39

CARLOTTO, M. S.; PALAZZO, L. S. Síndrome de Burnout e fatores associados: um estudo epidemiológico com professores. Caderno de Saúde Pública, Rio de Janeiro, v.22, n. 5, p. 1.017-1.026, maio 2006. Disponível em: http://www.scielo.br/pdf/csp/v22n5/14. pdf. Acesso em: 1 ago. 2017. http://dx.doi.org/10.1590/S0102-311X2006000500014

CARSON, K.; CARSON, P. P.; BEDEIAN, A. Development and construct validation of a career entrenchment measure. Journal of Occupational and Organizational Psychology, United Kingdom, v. 68, n. 4, p. 301-320, 1995. Disponível em: https://onlinelibrary.wiley.com/doi/abs/10.1111/j.2044-8325.1995.tb00589.x. Acesso em: 1 mar. 2011. https://doi.org/10.1111/j.2044-8325.1995.tb00589.x

CARVALHO, M. R. V. Perfil do professor da educação básica. Brasília, DF: Instituto Nacional de Estudos e Pesquisas Educacionais Anísio Teixeira, 2018. Disponível em: http://portal.inep.gov.br/informacao-da-publicacao/-/asset_ publisher/6JYIsGMAMkW1/document/id/1473981. Acesso em: 15 nov. 2018.

CASSETTARI, N.; SCALDELAI, V. F.; FRUTUOSO, P. C. Exoneração a pedido de professores: estudo em duas redes municipais paulistas. Educação \& Sociedade, Campinas, v. 35, n. 128, p. 909-927, jul./set. 2014. Disponível em: http://www.scielo.br/pdf/es/v35n128/0101-7330-es-35-128-00909.pdf. Acesso em: 7 mar. 2017. http://dx.doi.org/10.1590/ES0101-73302014096069

CENTRO DE ESTUDIOS MINEDUC. Evidencias: aportes a la reflexión sobre movilidad y abandono docente 2013-2014. Chile: Ministerio de Educación, 2015. p. 1-17. Disponível em: https://centroestudios.mineduc.cl/wp-content/uploads/ sites/100/2017/06/evidencia-final_enero2015.pdf. Acesso em: 12 jan. 2018.

CLEMENT, M. Why combatting teachers' stress is everyone's job. The Clearing House: A Journal of Educational Strategies, United Kingdom, v. 90, n. 4, p. 135-138, 2017. Disponível em: http://www.tandfonline.com/doi/full/10.1080/0 0098655.2017.1323519? scroll=top\&needAccess=true. Acesso em: 20 set. 2017. https://doi.org/10.1080/00098655.2017.1323519

COHEN, G.;BLAKE, R.; GOODMAN,D.Does turnover intention matter? Evaluating the usefulness of turnover intention rate as a predictor of actual turnover rate. Review of Public Personnel Administration, Thousand Oaks, v. 36, n. 3, p. 240-263, Sept. 2016. Disponível em: http://journals.sagepub.com/doi/pdf/10.1177/0734371X15581850. Acesso em: 20 set. 2017. https://doi.org/10.1177/0734371X15581850

COOPER, C.; DEWE, P.; O'DRISCOLL, M. Organizational stress: a review and critique of theory, research, and applications. Thousand Oaks, CA: Sage Publications, 2001. 
DICKTER, D. N.; ROZNOWSKI, M.; HARRISON, D. A. Temporal tempering: an event history analysis of the process of voluntary turnover. Journal of Applied Psychology, Washington, v. 81, n. 6, p. 705-716, Dec. 1996. Disponível em: http://psycnet.apa.org/fulltext/1996-06918-007.html. Acesso em: 1 mar. 2011. http://dx.doi.org/10.1037/0021-9010.81.6.705

DIEHL, L.; CARLOTTO, M. S. Conhecimento de professores sobre a síndrome de Burnout: processo, fatores de risco e consequências. Psicologia em Estudo, Maringá, v. 19, n. 4, p. 741-752, out./dez. 2014. Disponível em: http://www.scielo.br/pdf/pe/v19n4/1413-7372-pe-19-04-00741.pdf. Acesso em: 8 abr. 2017. http://dx.doi.org/10.1590/1413-73722455415

EUGÊNIO, B.; SOUZAS, R.; DI LAURO, A.D. Trabalho e adoecimento do professor da educação básica no interior da Bahia. Laplage em Revista, Sorocaba, v. 3, n. 2, p. 179-194, maio/ago. 2017. Disponível em: http://www.laplageemrevista.ufscar.br/index. php/lpg/article/view/325/493. Acesso em: 12 dez. 2017. https://doi.org/10.24115/ S2446-6220201732325p.179-194

FARIA, G. S. S.; RACHID, A. Jornada de trabalho dos professores da rede pública de ensino. Revista da FAE, Curitiba, v. 18, n. 2, p. 162-177, jul./dez. 2015. Disponível em: https://revistafae.fae.edu/revistafae/article/view/55/48. Acesso em: 12 dez. 2017. FIELD, A. Discovering statistics with SPSS. 2. ed. London: Sage Publications, 2009. FITCHETT,P.G.; LINEBACK, S.; MCCARTHY,C.J.; LAMBERT, R. G. Examining the relationship among teachers' working conditions, stress, and professional trajectory. In: PETTY, T.; GOOD, A.; PUTMAN, S. M. (ed.). Handbook of Research on Professional Development for Quality Teaching and Learning. Hershey, United States: IGI Global, 2016. p. 573-594.

FLEURI, R. M. Perfil profissional docente no Brasil: metodologias e categorias de pesquisas. Brasília, DF: Instituto Nacional de Estudos e Pesquisas Educacionais Anísio Teixeira, 2015. Disponível em: http://portal.inep.gov.br/documents/186968/486324/ Perfil+Profissional+Docente+no+Brasil+Metodologias+e+Categorias+de+Pesquisas/ 0265e3d7-3948-4b16-83c6-0b43dc14c6b3?version=1.2. Acesso em: 20 abr. 2018.

GIL-MONTE, P. R. El síndrome de quemarse por el trabajo (Burnout). Una enfermidade laboral en la sociedad del bienestar. Madrid: Pirâmide, 2005.

GRIMES, D. A.; SHULZ, K. F. An overview of clinical research: the lay of the land. The Lancet, London, v. 359, p. 57-61, Jan. 2002. Disponível em: https://www.ncbi.nlm.nih.gov/ pubmed/11809203.Acesso em:15 mar.2009.https://doi.org/10.1016/S0140-6736(02)07283-5 HONG, J. Y. Pre-service and beginning teachers' professional identity and its relation to dropping out of the profession. Teaching and Teacher Education, Amsterdam, v. 26, n. 8, p.1.530-1.543, Nov. 2010. Disponível em: https://eric.ed.gov/?id=EJ897501. Acesso em: 30 mar. 2016. https://doi.org/10.1016/j.tate.2010.06.003

HORVATH,M.; GOODELL,J.E.; KOSTEAS, V.D. Decisions to enter and continue in the teaching profession: evidence from a sample of US secondary STEM teacher candidates. Teaching and Teacher Education, Washington, DC, v. 71, p. 57-65, Apr. 2018. Disponível em: https://psycnet.apa.org/record/2018-12415-007. Acesso em: 12 fev. 2018. https://doi.org/10.1016/j.tate.2017.12.007 


\section{ILO - INTERNATIONAL LABOUR ORGANIZATION. Meeting of experts} on updating the list of occupational diseases. Geneva: ILO, 2005.

INGERSOLL, R. Teacher turnover and teacher shortages: an organizational analysis. American Educational Research Journal, Thousand Oaks,v.38,n.3,p.499-534,Jan.2001. Disponível em: http://journals.sagepub.com/doi/pdf/10.3102/00028312038003499. Acesso em: 19 mar. 2016. https://doi.org/10.3102/00028312038003499

INGERSOLL, R. Do we produce enough mathematics and science teachers? Phi Delta Kappan, Thousand Oaks, v. 92, n. 6, p. 37-41, Mar. 2011. Disponível em: http://journals.sagepub.com/doi/pdf/10.1177/003172171109200608. Acesso em: 30 mar. 2017. https://doi.org/10.1177/003172171109200608

INGERSOLL, R. Beginning teacher induction: what the data tell us. Phi Delta Kappan, Thousand Oaks, v. 93, n. 8, p. 47-51, May 2012. Disponível em: http://journals.sagepub.com/doi/pdf/10.1177/003172171209300811. Acesso em: 12 mar. 2017. https://doi.org/10.1177/003172171209300811

JEYARAJ, S. S. Occupational stress among the teachers of the higher secondary schools in Madurai district, Tamil Nadu. IOSR Journal of Business and Management, New York, v. 7, n. 5, p. 63-76, Jan./Feb. 2013. Disponível em: http://www.iosrjournals.org/iosr-jbm/papers/Vol7-issue5/I0756376.pdf. Acesso em: 1 abr. 2017. https://doi.org/10.9790/487X-0756376

KOROS, E.J.; MOMANYI,J.M.; CHAKUA, C. K. The impact of occupational stress on job satisfaction among Kenyan primary school teachers. International Journal of Scientific Research and Management, v. 6, n. 1, p. 51-56, Jan. 2018. Disponível em: http://ijsrm.in/index.php/ijsrm/article/view/1253. Acesso em: 30 jan. 2018. https:// doi.org/10.18535/ijsrm/v6i1.el08

LAPO, F. R.; BUENO, B. O. O abandono do magistério: vínculos e rupturas com o trabalho docente. Psicologia USP, São Paulo, v. 13, n. 2, p. 243-276, 2002. Disponível em: http:/www.scielo.br/scielo.php?pid=s0103-65642002000200014\&script=sci_ abstract\&tlng=pt. Acesso em: 25 jun. 2015. http://dx.doi.org/10.1590/S010365642002000200014

LEE, M.; GOODMAN, C.; DANDAPANI, N.; KEKAHIO, W. Review of international research on factors underlying teacher absenteeism. Washington: Department of Education, Institute of Education Sciences, National Center for Education Evaluation and Regional Assistance, Regional Educational Laboratory Pacific, 2015. Disponível em: https://ies.ed.gov/ncee/edlabs/regions/pacific/pdf/ REL_2015087.pdf. Acesso em: 30 nov. 2017.

LEE, T.; HOM, P.; EBERLY, M.; LI, J.; MITCHELL, T. On the next decade of research in voluntary employee turnover. Academy of Management Perspectives, New York, v. 31, n. 3, p. 201-221, June 2017. Disponível em: https://www.researchgate.net/ profile/Thomas_Lee60/publication/317311935_On_The_Next_Decade_of_Research_ in_Voluntary_Employee_Turnover/links/5a0a9ebfaca272d40f414118/On-The-NextDecade-of-Research-in-Voluntary-Employee-Turnover.pdf. Acesso em: 15 jan. 2018. https://doi.org/10.5465/amp.2016.0123 
LEITE, C. R.; LÖRH, S. S. Conflitos professor-aluno: uma proposta de intervenção. Revista Diálogo Educacional, Curitiba, v. 12, n. 36, p. 575-590, 2012. Disponível em: https://periodicos.pucpr.br/index.php/dialogoeducacional/article/view/4660.Acesso em: 12 maio 2019. http://dx.doi.org/10.7213/dialogo.educ.6101

LEMOS, J. C. G. Do encanto ao desencanto, da permanência ao abandono: o trabalho docente e a construção da identidade profissional. 2009. 315 f. Tese (Doutorado em Educação) - Pontifícia Universidade Católica de São Paulo, São Paulo. 2013.

LEMOS, J. C. G.; NOVAES, L. C. Juízos e práticas professorais na construção do processo de abandono do trabalho docente e o impacto sobre o trabalho pedagógico. Revista COCAR, Belém, v. 9, n. 18, p. 285-307, 2015. Disponível em: https://paginas.uepa.br/seer/index.php/cocar/article/view/714. Acesso em: 15 jan. 2018. LINDQYIST, P.; NORDÄNGER, U. K.; CARLSSON, R. Teacher attrition on the first five years - a multifaceted image. Teaching and Teacher Education, Amsterdam, v. 40, p. 94-103, May 2014. Disponível em: https://scinapse.io/papers/2021294211. Acesso em: 30 mar. 2017. https://doi.org/10.1016/j.tate.2014.02.005

MASLACH, C.; GOLDBERG, J. Prevention of Burnout: new perspectives. Applied and Preventive Psychology, Amsterdam, v. 7, n. 1, p. 63-74, 1998. Disponível em: https://psycnet.apa.org/record/1997-38974-004. Acesso em: 30 dez.2016. https://doi. org/10.1016/S0962-1849(98)80022-X

MATIJASCIC, M. Professores da educação básica no Brasil: condições de vida, inserção no mercado de trabalho e remuneração. Brasília, DF: Instituto de Pesquisa Econômica Aplicada, 2017. Disponível em: http://www.ipea.gov.br/portal/index. php?option=com_content\&view=article\&id=30338. Acesso em: 15 nov. 2018.

MEIRA, T. R. M.; CARDOSO, J. P.; VILELA, A. A.; AMORIM, C. R.; ROCHA, S. V.; ANDRADE, A. N.; FREIRE, D. S. Percepções de professores sobre trabalho docente e repercussões sobre sua saúde. Revista Brasileira em Promoção da Saúde, Fortaleza, n. 27, v. 2, p. 276-282, abr./jun. 2014. Disponível em: http:// periodicos.unifor.br/RBPS/article/view/2595/pdf. Acesso em: 15 jul. 2017. https:// doi.org/10.5020/18061230.2014.p276

MOBLEY, W. H. Intermediate linkages in the relationship between job satisfaction and employee turnover. Journal of Applied Psychology, New York, v. 62, n. 2, p. 237240, Apr. 1977. Disponível em: http://psycnet.apa.org/fulltext/1978-04582-001.pdf. Acesso em: 10 mar. 2015. https://doi.org/10.1037/0021-9010.62.2.237

MOBLEY,W.H.; HORNER, S.; HOLLINGSWORTH,A. An evaluation of precursors of hospital employee turnover. Journal of Applied Psychology, New York, v. 63, n. 4, p. 408-414, Aug. 1978. Disponível em: http://psycnet.apa.org/fulltext/1979-09964-001.pdf. Acesso em: 10 mar. 2015. https://doi.org/10.1037/0021-9010.63.4.408

NORONHA, M. M. B.; ASSUNÇÃO, A. A.; OLIVEIRA, D. A. O sofrimento no trabalho docente: o caso das professoras da rede pública de Montes Claros, Minas Gerais. Trabalho, Educação e Saúde, Minas Gerais, v. 6, n. 1, p. 65-86, mar. 2008. Disponível em: http://www.scielo.br/pdf/tes/v6n1/05.pdf. Acesso em: 15 set. 2017. http://dx.doi.org/10.1590/S1981-77462008000100005 
OECD - Organisation for Economic Co-Operation and Development. Teachers matter: attracting, developing and retaining effective teachers. Paris: OECD, 2005.

OECD - Organisation for Economic Co-Operation and Development. Talis 2013. Results: an international perspective on teaching and learning. Paris: OECD, 2014.

OLIVEIRA, D. A. A reestruturação do trabalho docente: precarização e flexibilização. Educação \& Sociedade, Campinas, v. 25, n. 89, p. 1.127-1.144, set./dez. 2004. Disponível em: http://www.scielo.br/pdf/es/v25n89/22614.pdf. Acesso em: 30 mar. 2010. http://dx.doi.org/10.1590/S0101-73302004000400003

PARDO MERINO, A.; RUIZ DÍAZ, M. A. SPSS 11: guía para el análisis de datos. Madrid: McGRAW-HILL, 2002.

PARSONSON, B. S. Evidence-based classroom behaviour management strategies. Kairaranga, New Zealand, v. 13, n. 1, p. 16-23, 2012. Disponível em: https://files.eric.ed.gov/fulltext/EJ976654.pdf. Acesso em: 15 jan. 2018.

PEREIRA JUNIOR, E. A.; OLIVEIRA, D. A. Indicadores de retenção e rotatividade dos docentes da educação básica. Cadernos de Pesquisa, São Paulo, v. 46, n. 160, p. 312-332, abr./jun. 2016. Disponível em: http://www.scielo.br/pdf/cp/ v46n160/1980-5314-cp-46-160-00312.pdf. Acesso em: 20 mar. 2017. http://dx.doi. org/10.1590/198053143370

PUCELLA, T. J. The Impact of National Board Certification on Burnout levels in educators. Clearing House: A Journal of Educational Strategies, Issues and Ideas, Philadelphia, v. 84, n. 2, p. 52-58, Feb. 2011. Disponível em: http://www.tandfonline.com/doi/full/10.1080/00098655.2010.511306. Acesso em: 1 jun. 2017. https://doi.org/10.1080/00098655.2010.511306

SALTINI, M. R.; VIDAL, A. G.; OLIVEIRA-SOBRINHO, A. S. Políticas públicas de educação e precarização do trabalho em São Paulo: o abandono da profissão docente na rede pública estadual. Trabalho e Educação, Belo Horizonte, v. 23, n. 1, p. 99-117, jan./ abr.2014. Disponível em: https://periodicos.ufmg.br/index.php/trabedu/article/view/9072. Acesso em: 12 maio 2019.

SHERNOFF, E. S.; MEHTA, T. G.; ATKINS, M. S.; TORF, R.; SPENCER, J. A qualitative study of the sources and impact of stress among urban teachers. School Mental Health, Washington, DC, v. 3, n. 2, p. 59-69, June 2011. Disponível em: https://link.springer.com/content/pdf/10.1007\%2Fs12310-011-9051-z.pdf. Acesso em: 20 mar. 2017. https://doi.org/10.1007/s12310-011-9051-z

SILVA, E. R.; ABUD, M. J. M. As representações docentes sobre as causas da indisciplina na escola: De quem é a culpa? Revista do GEL, São Paulo, v. 13, n. 1, p. 87-106, jan. 2016. Disponível em: https://revistas.gel.org.br/rg/article/view/548/0. Acesso em: 12 maio 2019. https://doi.org/10.21165/gel.v13i1.548

SILVA, L. B.; CUNHA, A. G. Experiências de trabalho de professores experientes da educação básica: qualidade de vida e esgotamento profissional. Psicologia e Saúde em Debate, Patos de Minas, v. 2, n. 2, p. 76-95, jan. 2017. Disponível em: http://psicodebate.dpgpsifpm.com.br/index.php/periodico/article/view/37/26. Acesso em: 20 dez. 2017. https://doi.org/10.22289/2446-922X.V2N2A5 
SILVA, O. G. S.; NAVARRO, E. C. N. A relação professor-aluno no processo ensinoaprendizagem. Interdisciplinar: Revista Eletrônica da Univar, n. 8, v. 3, p. 95-100, 2012. Disponível em: https://pt.scribd.com/document/247274539/A-RELACAO-PROFESSORALUNO-NO-PROCESSO-ENSINO-APRENDIZAGEM. Acesso em: 30 maio 2017. SING, N.; KATOCH, A. Study of occupational stress of secondary school teachers. International Journal of Advanced Education and Research, Delhi, India, v. 2, n. 4, p. 28-31, July 2017. Disponível em: www.alleducationjournal.com/ download/308/2-3-94-735.pdf. Acesso em: 20 jan. 2017.

SKAALVIK, E. M.; SKAALVIK, S. Job satisfaction, stress, and coping strategies in the teaching profession. What do the teachers say? International Education Studies, Ontario, v. 8, n. 3, p. 181-192, Feb. 2015. Disponível em: http://www.ccsenet.org/journal/index.php/ies/article/view/41245/24800. Acesso em: 20 mar. 2017. https://doi.org/10.5539/ies.v8n3p181

SPILT, J. L.; KOOMEN, H. M. Y.; THIJS, J. T. Teacher Wellbeing: The importance of teacher-student relationships. Educational Psychology Review, Switzerland, v. 23, p. 457-477, July 2011. Disponível em: https://research.vu.nl/ws/files/3137445/ Spilt\%20et\%20al\%202011\%20Teacher\%20Wellbeing.pdf. Acesso em: 15 nov. 2017. https://doi.org/10.1007/s10648-011-9170-y

TARTUCE, G. L. B. P.; NUNES, M. M. R.; DE ALMEIDA, P. C. A. Alunos do ensino médio e atratividade da carreira docente no Brasil. Cadernos de Pesquisa, São Paulo, v. 40, n. 140, p. 445-477, maio/ago. 2010. Disponível em: https://dialnet.unirioja.es/servlet/articulo?codigo=6208351. Acesso em: 10 abr. 2015. http://dx.doi.org/10.1590/S0100-15742010000200008

TEKLEAB, A. G.; TAKEUCHI, R.; TAYLOR, M. S. Extending the chain of relationships among organizational justice, social exchange, and employee reactions: The role of contract violations. Academy of Management Journal, New York, v. 48, n. 1, p. 146-157, Feb. 2005. Disponível em: http://amj.aom.org/content/48/1/146.full. Acesso em: 18 maio 2014. https://doi.org/10.5465/amj.2005.15993162

UNESCO - Organização das Nações Unidas para a Educação, a Ciência e a Cultura. Repensar a educação: rumo a um bem comum mundial? Brasília, DF: UNESCO, 2016. Disponível em: http://unesdoc.unesco.org/images/0024/002446/244670POR.pdf. Acesso em: 30 jan. 2017.

VEKEMAN, E.; DEVOS, G.; VALCKE, M.; ROSSEEL, Y. Do teachers leave the profession or move to another school when they don't fit? Educational Review, United Kingdom, v. 69, n. 4, p. 411-434, Sept.2016. Disponível em: http://www.tandfonline.com/ doi/full/10.1080/00131911.2016.1228610. Acesso em: 20 abr. 2017. https://doi.org/10.1 080/00131911.2016.1228610

WANG, C. K. J.; PYUN, D. Y.; KOH, K. T.; KWON, H. Harry. Teacher Burnout and teaching effectiveness in Singapore. Singapore: National Institute of Education, 2017. Disponível em: https://repository.nie.edu.sg/bitstream/10497/18639/1/NIE_research_ brief_16_013.pdf. Acesso em: 20 jan. 2018.

WOODS,P.Intensification and stress in teaching.In:VANDERBERGUE, R.;HUBERMAN, A.M. (eds.). Understanding and preventing teacher Burnout: a source book of international practice and research. Cambridge: Cambridge University Press, 1999. p. 115-138. 


\section{SOBRE AS AUTORAS}

Mary Sandra Carlotto é doutora em psicologia social pela Universidade de Santiago de Compostela (Espanha). Professora da Universidade do Vale do Rio dos Sinos (UNISINOS).

E-mail: mscarlotto@gmail.com

Sheila Gonçalves Câmara é doutora em psicologia pela Pontifícia Universidade Católica do Rio Grande do Sul (PUC-RS). Professora da Universidade Federal de Ciências da Saúde de Porto Alegre (UFCSPA). E-mail: sheila.gcamara@gmail.com

Michelle Engers Taube de Oliveira é mestre em psicologia clínica pela Universidade do Vale do Rio dos Sinos (UNISINOS). Professora da Universidade do Vale do Taquari (UNIVATES).

E-mail:michelletaube@hotmail.com

Recebido em 13 de março de 2018

Aprovado em 11 de março de 2019 